\title{
Model-Based Algebraic Approach to Robust Parameter Estimation in Uncertain Dynamics Rotating Machinery
}

\author{
Gerardo Pelaez, Pablo Izquierdo, Higinio Rubio, Manuel P. Donsion and Juan Carlos \\ Garcia-Prada
}

Department of Mechanical Engineering, University of Vigo (Pontevedra), Spain
Deptartment of Mechanical Engineering, University Carlos III, Leganes (Madrid), Spain

(Received 29 January 2016; accepted 28 October 2016)

This work presents a model-based algebraic approach to robust parameter estimation in uncertain dynamics rotating machinery. The approach evades some mathematical intricacies of the traditional stochastic methods, proposing an affordable Jeffcott-model-based scheme with several easy-to-implement computational advantages for processing a real-world rotor frequency response or orbit. Therefore, it takes out the dynamic parameters from one of the orbit's resonant humps when the multistage rotor orbit shape behaves closely to the Jeffcott-model orbit. This occurs for a valuable array of cases. The approach applies the spatial $\ell_{2}$-norm looking forward to the correlation between the analytical Jeffcott-orbit model and the experimental rotor's orbit hump, handling the normalized frequency ratio. Experimental results are also included to face this method with real-world rotating machinery orbits.

\section{INTRODUCTION}

Rotating machinery vibration response reflects the combined interaction of dynamic and stationary machine elements, in fact the history of rotodynamics consist in a collection of trade off between theory and practice dealing with the vibration response. There are situations where the experimental data of a case study rotor are unavailable due to some constraints. For instance, the machine running speed cannot be increased to investigate the detrimental effects of a resonant mode due to hazardous conditions. If this is the case, then modelling becomes the most appealing option. Modelling solutions include: Transfer Matrix Methods, Finite Element Analysis (FEA), and affordable analytical approachs. For the first and second cases, there are quite valuable computational plus time-cost penalties.

Alternatively, there is a wide array of the cases where there is a chance to develop rotor vibration field measurements under variable running speed. As an illustrative example, consider the pre-programmed shut downs and re-starts of a refinery unit in spite of the economic impact. If this is the case from a problem solving standpoint, then an investigation needs to be by comparing the predicted machine behaviour based on an affordable analytical model. The experimental measured behaviour then becomes the most appealing option. Thus, it is not surprising that a large amount of research and development has gone to deal with this subject as will be shortly disclosed in the following.

To understand the fundamental behaviour of a rotor, perhaps the first step should include determining the frequency of the system's critical speeds. Historically, one of the earliest procedures for critical speed calculations was developed by Julius Frith and Ernest Lamb (1901) dealing with torsional vibrations involved by brake of shaft with different inertial loads. ${ }^{1}$ However, in the beginning of the 1870th, transverse cracks occurred in the heavy horizontal rotors of some turbosets. This scenario stimulated the research on transverse vibrations in shafts. Rankine (1869) and Laval (1889) developed the primary theory corresponding to the determination of critical speeds by modeling flexible shafts with concentrated and distributed masses. ${ }^{2,3}$ A benchmark one degree of free- dom model proposal for an unbalanced rotating machine dates back to 1919, when Henry Hoffman Jeffcott developed a simple jet representative model based on a uniform flexible shaft over rigid bearings at its ends. ${ }^{4}$ The shaft mass plus the stage mass is concentrated at the shaft center. The Jeffcott rotor (named after Henry Homan Jeffcott), also known as the Gustaf de Laval rotor in Europe, is a simplified lumped parameter model. By this time 1920, the critical speeds of shafts with distributed masses were examined by Grammel. ${ }^{5}$ In the same period a discussion of shaft stability conditioned by internal friction forces was given by Kimball and Newkirk. ${ }^{6,7}$ Also, the turbine expert A. Stodola made an experimental analysis dealing with the gyroscopic effect of a disc mounted on a shaft and the secondary resonance phenomena. ${ }^{8}$ Nevertheless, the presence of disturbing forces is not restricted to unbalancing nature forces, in plain words a keyed shaft generates variable stiffness disturbing forces that excite resonance at angular velocities under the criticals. ${ }^{9}$

During the decades of 1940's and 1950's other similar cases were pointed out by Natanzon, Bogdanoff, Green and Dick. ${ }^{10}$ However, it was not until the next decade of 1960's when exhaustive analysis of problems relating to determination of natural vibration frequencies was developed. This analysis was given by Dimentber as a result of experiments carried out at the 'Elektrosila' Lab and the Leningrado Foundry. ${ }^{11}$ This is a more general problem than the determination of critical speeds because the latter are special frequency values of natural vibrations, which match the shaft speed of rotation at a given operation time. The valuable contribution of his hypotheses and propositions about shaft stability, verified experimentally, can be hardly emphasized at this point. By this time another notable contribution on the field thanks to Den Hartog, a mechanical engineer at Westhinghouse company, must be mentioned. ${ }^{9}$ Anyway, in all of these cases the shaft vibrations analyses is developed under a varying rotation speed.

During the 1980s, progress in analytical rotor modeling has been closely associated with the process of the transfer matrix calculations. On the other side, during 1980s, the rotating machinery monitoring, which was based on the signals suppield by sharp analog probes and digital keyphasors plus on- 
line digital signal processing, facilitated the progress of preventive fault detection and field balancing. To this goal, the rotor orbit, i.e., the log of transversal amplitudes of vibration plus phase delay of the deflective response, depicted in polar coordinates, reveals as a quite valuable tool as demonstrates the popular work of Donald Bently. ${ }^{12}$ Also, the primary works of Eisenman reinforce this notion. ${ }^{13} \mathrm{He}$ is a prolific author on the field of rotating machinery fault detection and balancing. At the 1990th, Eisenman's son got an application to work as manager of rotating equipments at a refinery. He came back home almost every day carrying a question on the subject for his father Eisenman Sr. The answers to the questions became the main argument of a new textbook written by both. ${ }^{14}$ Anyway, in most of the aforementioned works the rotor orbit shape becomes paramount to examine the shaft vibrations nature resulting form varying rotation speed. Between others the orbit shape allows to characterize cracked or bowed shafts, mechanical looses, i.e., machinery malfunctions.

However, the estimation of the frequency corresponding to system critical speeds from the orbit requires a more detailed analysis than the only consideration of the orbit shape referred above. In fact, the exact frequency at which it occurs has a rather valuable uncertainty associated due to the fast phase variation at such resonant region. Overcome this drawback become one of the arguments for the present work.

By the 21st century, a wide array of works have been published which deal with the stability analysis and control of unbalanced shafts vibrations. The stability analysis of the Laval's rotor with a transverse crack was developed. ${ }^{15}$ Vibrations isolation with a semi-active pneumatic damping was also presented. ${ }^{16}$ Auto-balancing using speed dependent vibration absorbers was also investigated. ${ }^{17}$ Radial magnetic force was used for shaft vibration suppression to go through the first critical speed. ${ }^{18}$ Under a periodic axial force, a rotating Timoshenko shaft with a rigid unsymmetrical disc was modelled as a parametrically excited system using the finite-element method and a harmonic balance method. ${ }^{19}$ Undesired whirling motions of rotating machines are efficiently reduced by supporting journal boxes elastically and controlling their movement by viscous dampers or by dry friction surfaces normal to the shaft axis. $^{20}$ Damping lateral vibrations using rotor speed modulation also become a recent alternative presented. ${ }^{21}$ The method is based on the generation of an harmonic additive to the constant rotation speed, that provides significant damping at criticals. This suggests the notion that the influence of the electric drive-converter can not be neglected dealing with real-world rotating machines powered by electric motors plus converters. This issue is taking into account in the present work, by installing a well suited passive LC filter behind a three phase PWM driver. This set up guarantees that a sinusoidal modulation feed the asynchronous electrical motor minimizing harmonics detrimental effects in the experimental orbits.

The present work is organized as follows: in section- 2 symbolic computations, involving the normalized frequency ratio, over the analytical Jeffcott-Orbit expression, are developed. These will facilitate in section-3 synthesize an advantageous analytical formulation, regarding the orbit extreme points properties. Thus, the estimation of the actual critical frequency on the basis of the correlation between the experimental multi-stage rotor orbit-hump to the equivalent Jeffcottmodel-rotor orbit by Least Squares Fitting algebraic method. Later, the synthesized analytical formulation to estimate the corresponding Jeffcott-model-rotor parameters was applied: stiffness of the shaft, damping ratio and the unbalance force phase. In section 4 , experimental results dealing with a real-

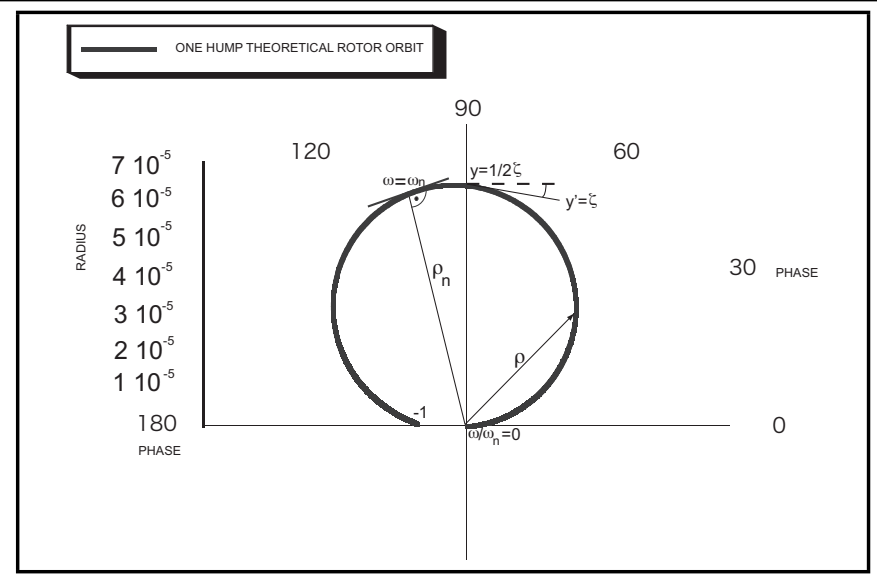

Figure 1. Jeffcott-model rotor orbit. Conditions: $\omega_{n}=23.56 \mathrm{~Hz}, \zeta=0.1$.

world multi-stage rotor plus continuos and keyed shafts, are given. Also, a discussion on the aforementioned parameter estimation good performance. Finally, section-5 includes some modest conclusions.

\section{THE MODEL}

Dealing with the wide array of machines with rolling element bearings without resilience at the shaft journals, usually synchronous whirling takes place. In plain words, the flexible shaft and the inertial stages whirls with the same angular velocity, so that rotate together as a rigid body. Also, if the inertial stages are close to the shaft center and the customary range of rotating velocities behaves moderate, the gyroscopic effects can be assumed moderate as well. If this the case, the resonant hump shape of a multi-stage rotating machine orbit, behaves closely to the orbit shape of a Jeffcott-model equivalent machine. ${ }^{22}$

Regarding the conditions stated above, the stationary frequency response of a flexible shaft without resilience at the journals and the inertial stage close to the shaft center has been exhaustively addressed in the the literature ${ }^{11},{ }^{22}$ and is given by

$$
X(i \omega)=\frac{\omega_{n}^{2} e_{u}}{\omega^{2}-\omega_{n}^{2}+2 \zeta \omega \omega_{n} i}=\frac{e_{u}}{\left.\left(\left(\frac{\omega}{\omega_{n}}\right)^{2}-1\right)\right)+i 2 \zeta \frac{\omega}{\omega_{n}}} .
$$

Nonetheless, an interesting geometric translation can be extracted from this expression given in terms of the normalized frequency ratio. To this goal, let it be $X(i \omega)=x-i y$ a customary complex number, $q=\frac{\omega}{\omega_{m}}$ the normalized frequency a non-dimensional speed of rotation that states the frequency ratio between the actual rotating frequency and the assumed or modeled natural frequency. This normalized value q, is usually adopted for robust filters design. ${ }^{23}$ Note that the value anticipated by the referred benchmark model for the natural frequency would be $\omega_{n} \simeq \omega_{m}=\sqrt{\frac{k}{m}}$ where $k=48 E I_{z} / \ell^{3}$ and $m$ states the mass of the inertia disk. Finally, without lost of generality $e_{u}$ is allowed to be the unit, then Eq. (1) becomes

$$
x-i y=\frac{1}{\left(q^{2}-1\right)+2 \zeta q i} .
$$

The use of algebraic manipulations to expand Eq. (2) as a function of the normalized frequency ration $q$ gives

$$
\begin{aligned}
& x\left(q^{2}-1\right)+2 \zeta y q=1 ; \\
& 2 \zeta x q-\left(q^{2}-1\right) y=0 .
\end{aligned}
$$




\begin{tabular}{l}
\hline \hline Table 1. Jeffcott Model Concerned Parameters. \\
\begin{tabular}{|l|r|}
\hline Machine model parameter & Assumed value \\
\hline Young module, $E$ & $206,000 \mathrm{MPa}$ \\
\hline Rotor Diameter, $d$ & $0.015 \mathrm{~m}$ \\
\hline Second moment of the cross section, $I_{z}$ & $\left(\pi d^{4} / 64\right) 2.48 \cdot 10^{-9} \mathrm{~m}^{4}$ \\
\hline Rotor length, $l$ & $0.500 \mathrm{~m}$ \\
\hline Unbalanced distance, $e_{u}$ & $0.010 \mathrm{~m}$ \\
\hline Asynchronous motor nominal velocity & $1500 \mathrm{rpm}$ \\
\hline Disk mass, $m$ & $9 \mathrm{Kgr}$. \\
\hline
\end{tabular}
\end{tabular}

Eliminating the parameter q between Eq. (3) and Eq. (4) can be done by firstly rewriting them as

$$
\begin{gathered}
\left(q^{2}-1\right)=\frac{1-2 \zeta y q}{x} \\
\left(q^{2}-1\right)=\frac{2 \zeta x q}{y}
\end{gathered}
$$

it follows that

$$
y-2 \zeta y^{2} q=2 \zeta x^{2} q
$$

therefore

$$
q=\frac{y}{2 \zeta\left(x^{2}+y^{2}\right)}
$$

Substituting the $q$ parameter value given by Eq. (8) into $\mathrm{Eq}(3)$ yields

$$
\begin{aligned}
x y^{2}-x\left(2 \zeta\left(x^{2}+y^{2}\right)\right)^{2}+2 \zeta y^{2}+ & 2 \zeta y^{2} 2 \zeta\left(x^{2}+y^{2}\right) \\
& =\left(2 \zeta\left(x^{2}+y^{2}\right)\right)^{2}
\end{aligned}
$$

reordering terms and rejecting the null solution, Eq. (9) becomes

$$
y^{2}-4 \zeta^{2} x^{4}-8 \zeta^{2} x^{2} y^{2}-4 \zeta^{2} y^{4}-4 \zeta^{2} y^{2} x-4 \zeta^{2} x^{3}=0 .
$$

At this point, acknoledging that $x^{2}<<y^{2}$, rejecting second order terms closed to zero Eq. (10) yields

$$
y^{2}-4 \zeta^{2} y^{4}-4 \zeta^{2} y^{2} x \simeq 0 .
$$

This a quadratic equation in $y$, again rejecting the trivial solution it simply becomes

$$
1 \simeq 4 \zeta^{2}\left(y^{2}-x\right)
$$

The Eq. (12) expresses a parabola curve whose hump intersection with the y-axis is at the point $y=\frac{1}{2 \zeta}$. Differentiating Eq. (12), the slope value at such point is $\dot{y}=\zeta$, depicted in Fig. 1. Thus, using this property, it is feasible to extract the damping ratio from the theoretical rotor orbit. Also accordingly, the orbit hump size is a function of the damping ratio. In general terms, as the damping increases the orbit size reduces. Anyway this is only one of the outcomes that can be obtained from the orbit geometric properties. In fact, the orbit hump provides quite valuables tracks in order to analyse the dynamic behaviour of the rotor.

\section{METHODS}

Actually, dealing with a real world rotating machine orbit, several humps might take place. Each one corresponds to a resonant situation. All of them lumped together build the whole rotating machine orbit. In addition, each hump has its own orientation in the complex plane. Thus, between each hump of a real world rotating machine orbit and the Jeffcott-model orbit, depicted in Fig. 2, the following differences can be found:

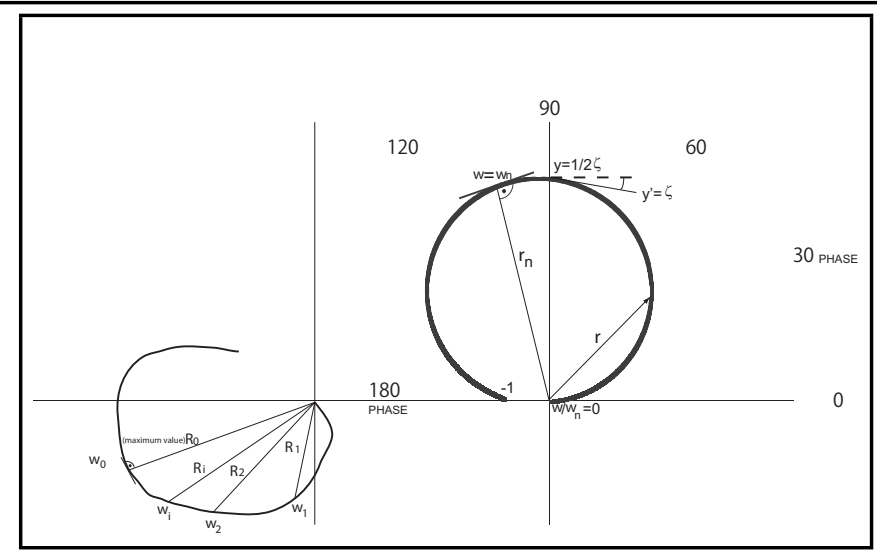

Figure 2. Sketch for comparison of the actual orbit and the Jeffcott-model orbit.

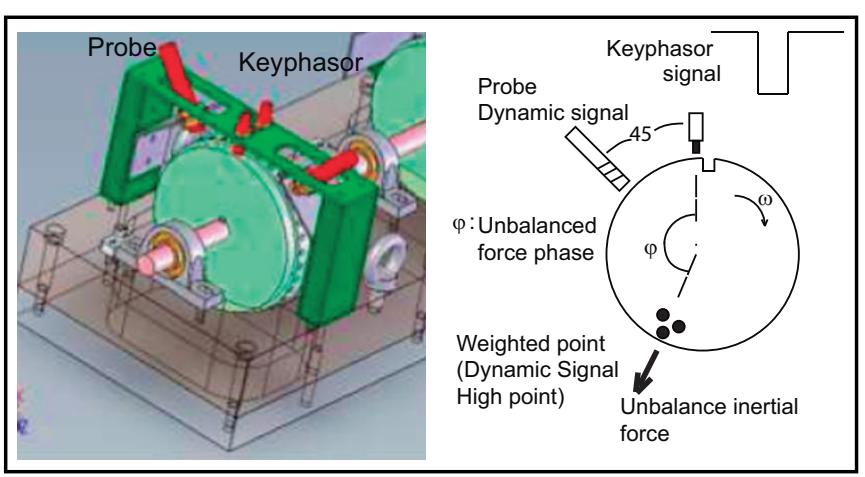

Figure 3. Analog Probe plus digital Keyphasor layout at the measurement bridge.

1. The parameters $\rho$ and $q$ involved by the Jeffcott-model orbit are dimensionless while the real world hump values: $R$ the amplitude, and $\omega$ the frequency have dimensions.

2. With reference to the initial and final points of the Jeffcott-model orbit, the actual orbit lacks such points or regions corresponding to the cases when $\omega$ equals zero and $\omega$ equals $\infty$, which can not be reached.

3. In fact, each hump of the orbit is not placed at the theoretical orientation, its origin is arbitrary and it is a function of other humps that the actual orbit might have.

4. Finally, the shape of an orbit's hump would differ from the Jeffcott-model orbit.

Anyway, this work attempts to overcome these drawbacks. Unless otherwise stated the availability of experimental reliable measurements corresponding to the rotor deflection $R_{i}$ at the frequency $\omega_{i}$, are quite valuable. The maximum deflection $R_{0}$ of the orbit hump is a reliable value, however the exact frequency at which it occurs $\omega_{n}$ versus $\omega_{0}$ have a quite valuable uncertainty associated due to the fast phase variation at such resonant region. Actually, the most affordable experimental approach consist in measure the inertial unbalanced force phase depicted in Fig. 3. Two signal are involved: the periodic signal supplied by the keyphasor plus the analog signal supplied by the probe. For a given rotor angular velocity such angular phase, keeping unchanged the angular velocity, remains proportional to the time delay between the analog signal high point, corresponding to inertial stage weighted point, and the keyphasor flank as shows the Fig.4

The experimental measurement outcomes resulting from the just described set up reinforces the notion of the fast phase 


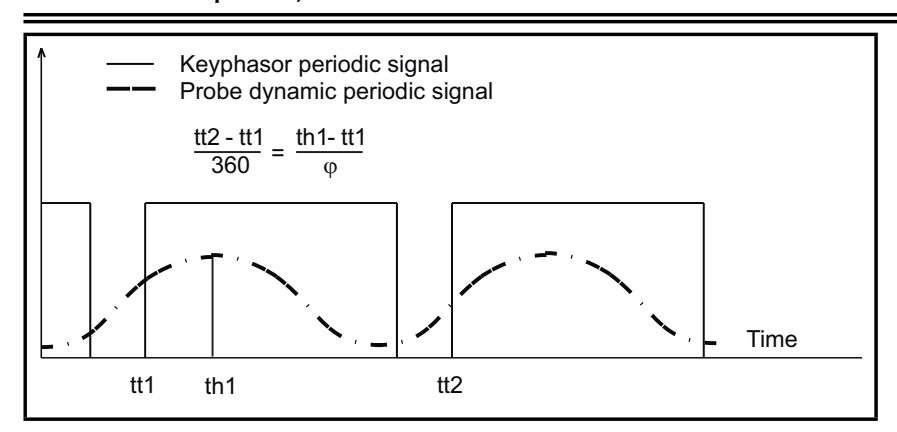

Figure 4. Time Domain Output signals: Keyphasor plus probe dynamic periodic signals.

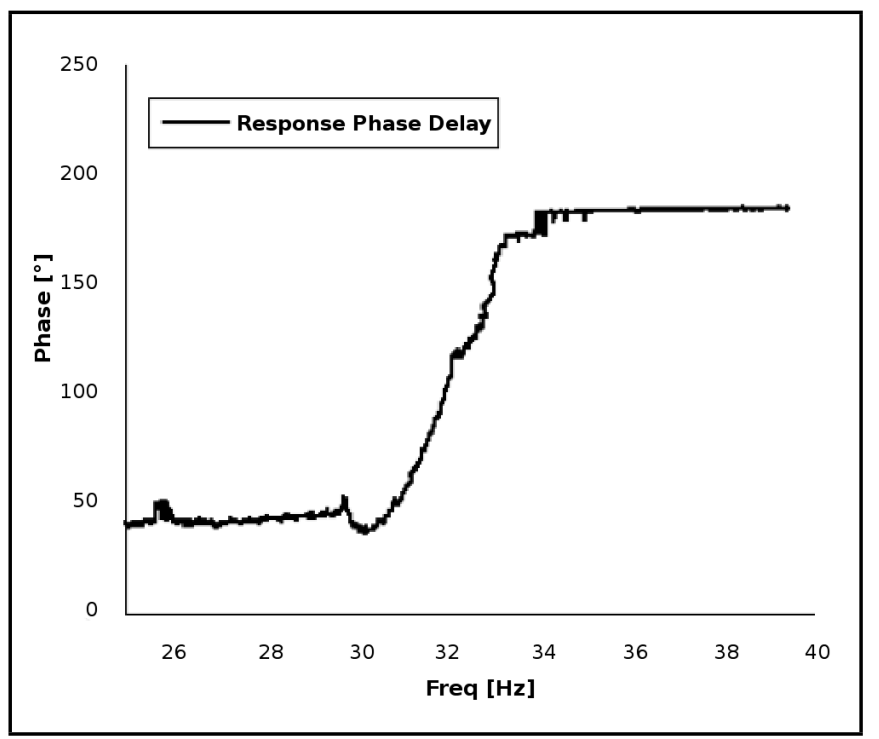

Figure 5. The phase variation at the resonant region (experimental data).

variation corresponding to the inertial force when the rotor traverses the critical, as demonstrated overall in Fig. 5. In view of this, to estimate the actual critical frequency $\omega_{n}$ of a multistage rotating machine from an experimental orbit-hump at a resonant zone, the following analysis is developed.

First, recall from Eq. (2) that the theoretical amplitude of vibration $\rho$ can be expressed by

$$
\rho=\frac{1}{\sqrt{\left(q^{2}-1\right)^{2}+4 \zeta^{2} q^{2}}} .
$$

Let it be $\rho_{0}$ the maximum dimensionless rotor deflection amplitude that takes place at the normalized frequency ratio $q_{0}$. If this the case the condition that occurs, at this extreme, is

$$
\left[\frac{d \rho}{d q}\right]_{0}=0 .
$$

From this point, Maple 13 was used to carry out the values of $\rho_{0}$ and correspondingly $q_{0}$ plus other symbolic computations. Deriving Eq. (13) according Eq. (14) it is first obtained

$$
q_{0}= \pm \sqrt{1-2 \zeta^{2}}
$$

by simply substituting this value of $q_{0}$ as a function of $\zeta$, versus Eq. (15) into Eq. (13) yields,

$$
\rho_{0}=\frac{1}{\left(1-2 \zeta^{2}\right) \sqrt{\left(1-\frac{1}{1-2 \zeta^{2}}\right)^{2}+\frac{2 \zeta^{2}}{1-2 \zeta^{2}}}} .
$$

In addition and inversely, by using symbolic computation any dimensionless amplitude of vibration $\rho$ can be expressed in terms of its corresponding normalized frequency ratio $q=\frac{\omega}{\omega_{m}}$ plus $q_{0}=\frac{\omega_{0}}{\omega_{m}}$, without involving the damping ratio $\zeta$ parameter.

$$
\rho=\frac{1}{q^{2} \sqrt{\frac{1}{q^{4}}-\frac{2 q_{0}^{2}}{q^{2}}+1}} .
$$

Next, this result was used to solve the correlation between an experimental rotor orbit and the theoretical one synthesized above on the basis of the assumed or modeled natural frequency $\omega_{m}$ and damping ratio $\zeta_{m}$.

Estimation of the actual critical frequency on the basis of the experimental orbit correlation with the Jeffcott-model orbit. At this point, the experimental rotor orbit will be used to extract the actual critical frequency, that coincides with a natural frequency under the resonant situation. To this end, as shown in Fig. 2, let it be the ratio between any experimentally measured amplitude of vibration $R_{i}$ and the maximum measured amplitude $R_{0}$. Note that $R_{0}$ is a reliable value and able to be easily obtained from the experimental measurements

$$
\frac{R_{i}^{2}}{R_{0}^{2}}=\frac{1}{Z_{i}}
$$

where the dimensionless parameter $Z_{i}>1$ states the inverse of such ratio. And for each frequency $\omega_{i}$ corresponding to the amplitude $R_{i}$, let it be

$$
b_{i}=\frac{\omega_{i}^{2}-\omega_{0}^{2}}{\omega_{0}^{2}}
$$

the squared normalized $\ell^{2}$-norm. Recalling that $q=\omega / \omega_{m}$ states again the normalized frequency. Thus, dividing the second's term, num and den, of Eq. (19) by $\omega_{m}^{2}$ is equivalent to

$$
q_{i}^{2}=q_{0}^{2}\left(1+b_{i}\right)
$$

where $\omega_{0}$ states the actual global machine critical frequency. Looking forward, the correlation between the experimental and the theoretical orbit yields

$$
\frac{R_{1}}{\rho_{1}}=\frac{R_{2}}{\rho_{2}}=\cdots=\frac{R_{i}}{\rho_{i}}=\cdots=\frac{R_{0}}{\rho_{0}} ;
$$

thus, according to Eq. (18) and (21) it can be written

$$
\frac{R_{i}^{2}}{R_{0}^{2}}=\frac{\rho_{i}^{2}}{\rho_{0}^{2}}=\frac{1}{Z_{i}} ;
$$

where $Z_{i}=\frac{R_{0}^{2}}{R_{i}^{2}}$ is a dimensionless scalar. At this point, substituting Eq. (17) into Eq. (22) yields,

$$
\frac{\rho_{i}^{2}}{\rho_{0}^{2}}=\frac{1-2 q_{0}^{4}+q_{0}^{2}}{1-2 q_{0}^{2} q_{i}^{2}+q_{i}^{2}}
$$

combining Eq. (23), (22), and (20) gives,

$$
\frac{1}{Z_{i}}=\frac{1-q_{0}^{4}}{1-q_{0}^{4}+q_{0}^{4} b_{i}^{2}} ;
$$

rearranging terms, Eq. (24) becomes

$$
b_{i}^{2}=\left(Z_{i}-1\right) \cdot \frac{1-q_{0}^{4}}{q_{0}^{4}} ;
$$




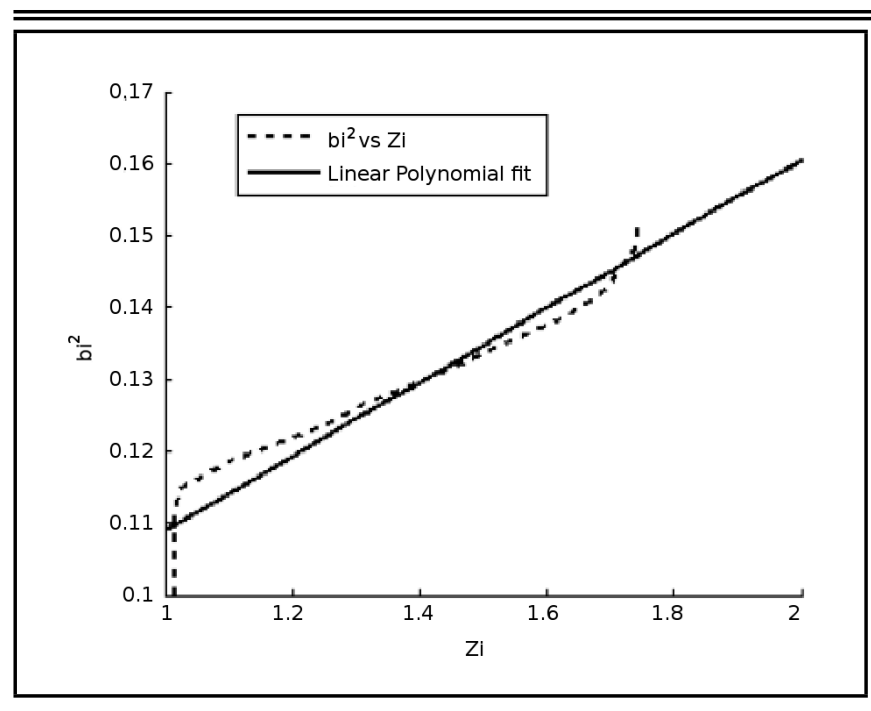

Figure 6. The Least Square polynomial fitting for the set of points $\left(b_{i}^{2}, Z_{i}\right)$.

finally, letting

$$
Q=\frac{1-q_{0}^{4}}{q_{0}^{4}}
$$

where $q_{0}=\frac{\omega_{0}}{\omega_{m}}$. Thus the Eq. (25) can be simply rewritten as

$$
b_{i}^{2}=Q \cdot\left(Z_{i}-1\right)
$$

A linear relationship between $b_{i}^{2}$ and $Z_{i}$ has been found. Such linear polynomial traverses the point $(1,0)$ of the $Z i-b i^{2}$ plane. In addition, according to Eq. (26), its slope Q links the prime concern modelled frequency $\omega_{m}=\sqrt{\frac{k}{m}}$ of the Jeffcott model ( $\omega_{m}=23.46[\mathrm{~Hz}]$ according to table 1 ), and the actual global machine critical frequency, $\omega_{0}$. However, given a set of experimental points $\left(Z_{i}, b_{i}^{2}\right)$ coming from the measured response in the frequency domain configuring the rotating machine orbit, there is a mismatch between this set of experimental points $\left(Z_{i}, b_{i}^{2}\right)$ and the theoretical linear polynomial found.

In view of this scenario, the most appealing option comprises a Least Square polynomial fitting procedure shown in Fig. 6. The first step consists in building a table of the type shown in Table 2. Given the dataset $\left(Z_{i}, b_{i}^{2}\right)$ the method of least squares minimizes the error associated to saying $b_{i}^{2}=$ $Q \cdot\left(Z_{i}-1\right)$ by

$$
\min \rightarrow E(Q)=\sum_{i=1}^{3}\left(b_{i}^{2}-Q \cdot\left(Z_{i}-1\right)\right)^{2} .
$$

To this goal, it is necessary the availability of an initial modelled or guess frequency $\omega_{m}$. Recall that $Q$ is a function of $q_{0}$ and $q_{0}$ is a function of $\omega_{m}$ because $q_{0}=\frac{\omega_{0}}{\omega_{m}}$. However, for such a guess frequency, the outcoming line obtained from the fitting process, must traverse the point $(1,0)$. If this constraint is not verified, the value of the assumed modeled frequency $\omega_{m}$ must be shifted upwards or downwards by the algebraic algorithm until the linear polynomial traverses the constraint point as shown in Fig. 7 and Table 3. Once a well suited $\omega_{m}$ value has been reached from the least squares method, the slope Q of the corresponding linear polynomial allows to carry out $\omega_{0}$ using

$$
\omega_{0}=\omega_{m} \frac{1}{\sqrt[4]{1+Q}} .
$$

Table 2. Experimental Measurements Involved Ratios.

\begin{tabular}{|c|r|r|r|r|}
\hline i-input & $R_{i}$ & $\omega_{i}$ & $b_{i}^{2}=\left(\frac{\omega_{i}^{2}-\omega_{0}^{2}}{\omega_{0}^{2}}\right)^{2}$ & $Z_{i}=\frac{R_{0}^{2}}{R_{i}^{2}}$ \\
\hline 1 & 10.13 & 26.4061 & 0.0315 & 1 \\
\hline 2 & 10.13 & 26.441 & 0.0342 & 1 \\
\hline 3 & 10.0789 & 26.476 & 0.037 & 1.0102 \\
\hline 4 & 10.0782 & 26.5252 & 0.0408 & 1.0103 \\
\hline 5 & 10.0747 & 26.5393 & 0.0419 & 1.011 \\
\hline 6 & 10.0785 & 26.5887 & 0.0458 & 1.0102 \\
\hline 7 & 10.0805 & 26.6241 & 0.0486 & 1.0099 \\
\hline 8 & 10.0779 & 26.6667 & 0.0519 & 1.0104 \\
\hline 9 & 10.0769 & 26.7094 & 0.0553 & 1.0106 \\
\hline 10 & 10.0776 & 26.7237 & 0.0564 & 1.0104 \\
\hline 11 & 10.0798 & 26.7666 & 0.0598 & 1.01 \\
\hline
\end{tabular}

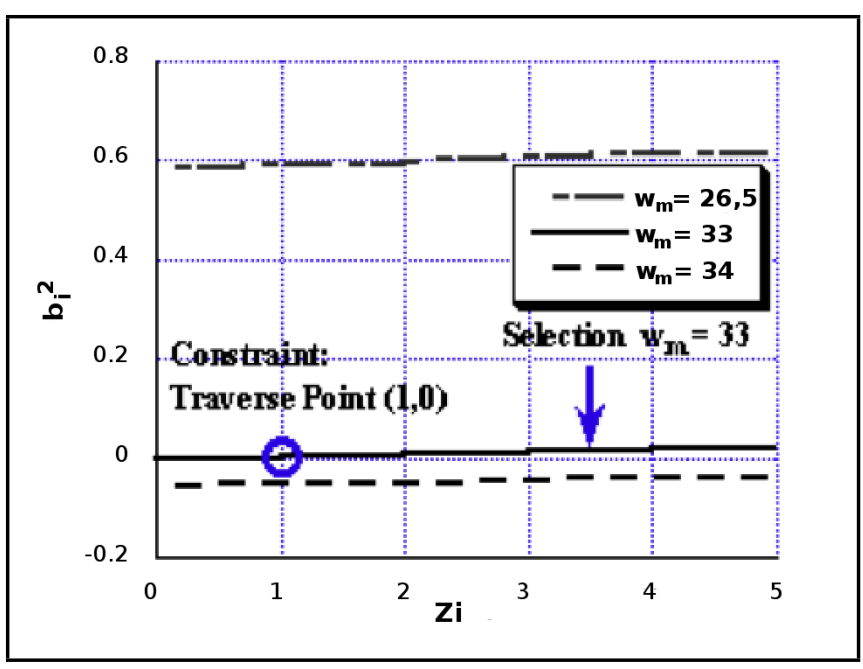

Figure 7. Convergence of the $\mathrm{LQE}$ linear polynomial fit procedure. Constrain: traverse the point $(1,0)$ of the $Z i-b i^{2}$ plane.

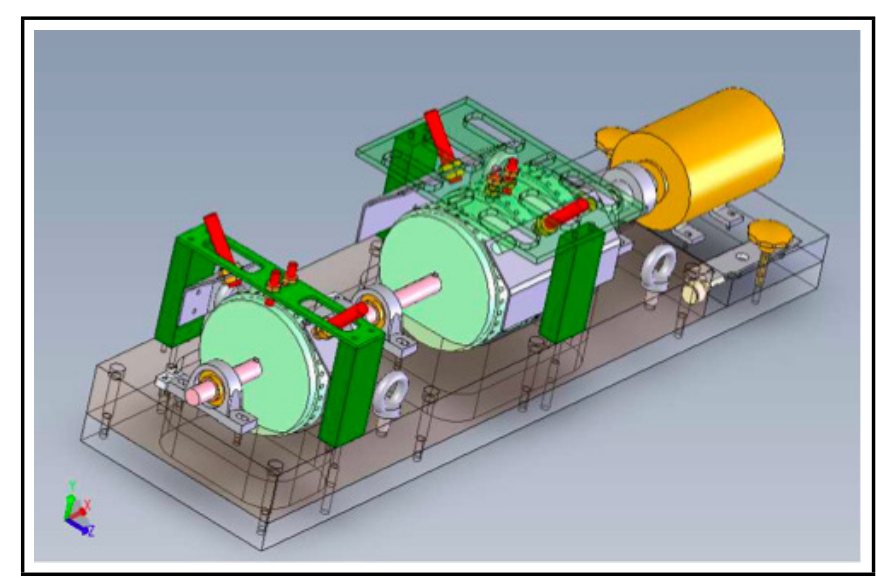

Figure 8. Experimental set up.

Shortly, the procedure just described provides an affordable method for evaluating the corresponding critical frequency of an orbit hump. The relevance of this procedure can be stated by recalling that the exact frequency at which it occurs $\omega_{n}$ versus $\omega_{0}$ have a quite valuable uncertainty associated due to the fast phase variation at such resonant hump-region.

However, this is only the primary result. In addition, the presented method might be helpful to identify others dynamics parameters. To that goal let assume that the mass value of the inertial stage is known, this is a quite reasonable supposition. 
Table 3. Convergence of the LQE linear polynomial fit procedure.

\begin{tabular}{|l|l|l|r|}
\hline$\omega_{m}$ & linear polynomial & $Z_{i}$ & $b_{i}^{2}$ \\
\hline 26.5 & $b_{i}^{2}=0.006684 Z_{i}+0.5853$ & 1 & 0.591984 \\
\hline 33 & $b_{i}^{2}=0.004616 Z_{i}+0.0002295$ & 1 & $\gg 0.0048455$ \\
\hline 34 & $b_{i}^{2}=0.004348 Z_{i}-0.05774$ & 1 & -0.0533952 \\
\hline
\end{tabular}

Thus, from Eq. (26) the shaft stiffness can be obtained as,

$$
k=\omega_{0}^{2} m \sqrt{1+Q} .
$$

The damping ratio can be easily obtained from Eq. (15)

$$
\zeta=\sqrt{\frac{1-q_{0}^{2}}{2}}
$$

Or equivalently identify the damping of the benchmark model

$$
c=\sqrt{k m\left(1-q_{0}^{2}\right)}
$$

Dealing with the phase delay of the shaft response to the unbalance force $F$ at the system critical $\omega_{0}$, it is given by

$$
\tan \left(\varphi_{0}\right)=\frac{2 \zeta q_{0}}{1-q_{0}^{2}}
$$

The direction of $\rho_{0}$ can be found into the orbit, in view of this, from Eq. (33) the direction of the unbalance excitation force $F$, that causes the rotor orbit, can be also estimated as shown in Fig. 9. Finally, the module of $F$ can be found because

$$
R_{0} k=F \rho_{0} .
$$

Thus, from Eq. (17) and Eq. (33) it is obtained

$$
F=k R_{0} \sqrt{1-q_{0}^{4}}
$$

At this point, the procedure just described above allows to estimate the corresponding Jeffcott-model parameters from one of the orbit's resonant humps, when the multi-stage rotor orbit shape behaves closely to the Jeffcott-model orbit. If, as it is customary, the mass $m$ is the parameter whose value is available then the a simple look up method was given above to estimate the addressed model parameters.

\section{MATERIALS}

\subsection{Continuous Shaft Rotor}

To demonstrate these assessments, a multi-stage rotating machine experimental response to a varying velocity was collected. The sketch of the machine is shown in Fig. 8. The machine is composed by two inertial stages with associated measurement planes consisting of a bridge where two analog probes plus a digital keyphasor have been installed. Table 1 includes the theoretical parameters corresponding to the modeled shaft stiffness plus individual disk inertia, when the two stages are coupling to the asynchronous motor. Finally, the frequency response bellow the second critical frequency is shown in Fig. 9. This orbit was translated to the $Z_{i}-b_{i}^{2}$ plane depicted in Fig. 10. This dataset underwent the Least Square fitting procedure with the help of MATLAB $\cap$ Toolstrip. The MATLABscript procedure converges to a solution for $\omega_{m}=33 \mathrm{~Hz}$ as demonstrates the Fig. 7. Also, the slope of the optimal linear polynomial found was $\mathrm{Q}=0.004616 \pm 8.3510^{-5}$. Thus,

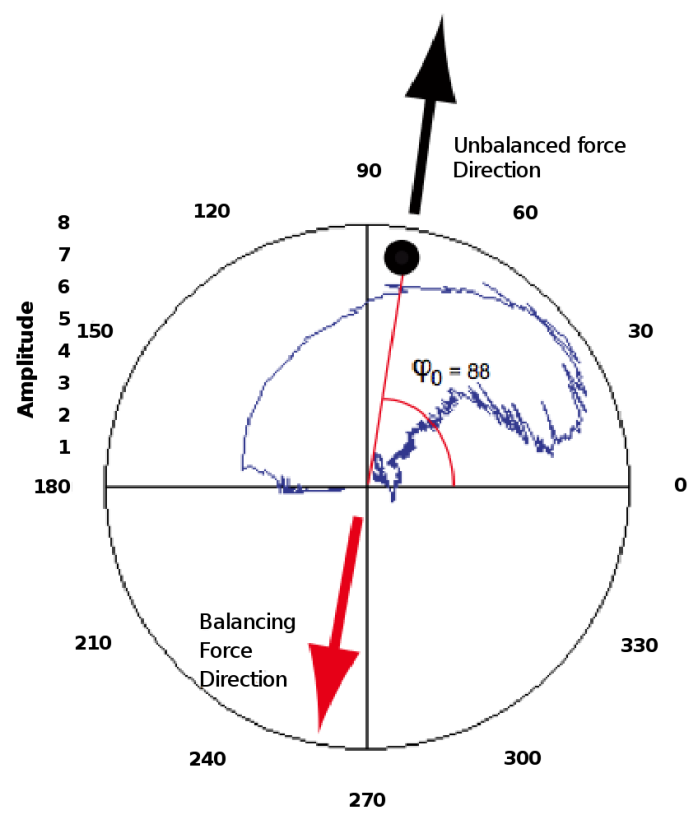

Figure 9. Unbalanced rotor experimental orbit. Conditions: linear response.

the actual critical frequency estimated according Eq. (28) was $\omega_{0}=32.962 \pm 7 \cdot 10^{-4} \mathrm{~Hz}$, carrying a low level of uncertainty associated, as shows the figure 11 .

$$
\begin{aligned}
& \omega_{0}=33 \frac{1}{\sqrt[4]{1+0.004616 \pm 8.35 \cdot 10^{-5}}} \\
& \quad=32.962 \pm 7 \cdot 10^{-4} \mathrm{~Hz} .
\end{aligned}
$$

To verify this result, a finite element model of the corresponding Jeffcott-model rotor plus the electrical motor was built, as shown in Fig. 12, giving a first natural frequency of around $44[\mathrm{~Hz}]$. Regarding that, in general terms, the FEM method is an extension of the Rayleigh's energy approximation method, ${ }^{24}$ it is customary to obtain for the lowest natural frequency a value which is somewhat quite high. ${ }^{9}$ This result reinforces this notion, when compared with the experimental value obtained above, extracted from the actual real world multi-stage rotor orbit.

Assuming that the mass $m$ is the parameter whose value is known given in table 1, the corresponding Jefcott-model shaft stiffness can be estimated according to Eq. (29),

$$
\begin{array}{r}
k=\omega_{0}^{2} m \sqrt{1+Q}=(32.962 * 2 \pi)^{2} \cdot 9 \cdot \sqrt{1+0.004616} \\
=3.87 \cdot 10^{5} \mathrm{~N} / \mathrm{m} .
\end{array}
$$

The modeled value for the shaft stiffness were $k_{m}=\frac{48 E I_{z}}{\ell^{3}}=$ $1.96 \cdot 10^{5} \mathrm{~N} / \mathrm{m}$ according to Table 1 . The estimated value found for the stiffness differs largely from the beam-model one, actually it is nearly twice the initially modelled value. The procedure also allows to estimate the damping ratio value. According to Eq. (30), it is given by

$$
\zeta=\sqrt{\frac{1-q_{0}^{2}}{2}}=\sqrt{\frac{1-\left(\frac{32.96}{33}\right)^{2}}{2}}=0.0335 ;
$$




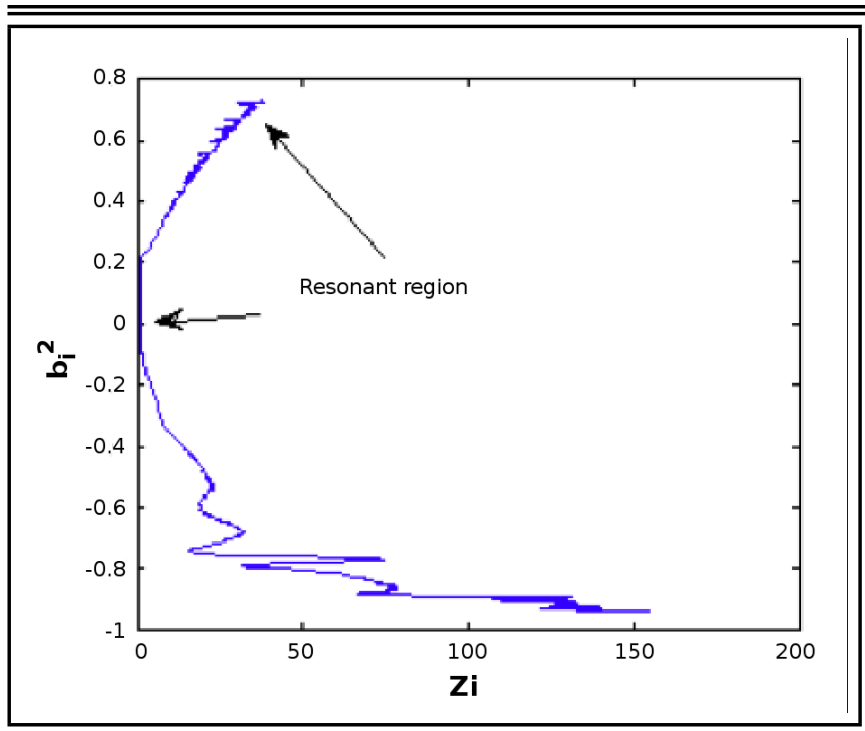

Figure 10. Transformation of the orbit into the $Z i-b_{i}^{2}$ plane.

or equivalently the damping

$$
\begin{array}{r}
c=\sqrt{k m\left(1-q_{0}^{2}\right)}=\sqrt{3.87 \cdot 10^{5} \cdot 9 \cdot\left(1-\left[\frac{32.96}{33}\right]^{2}\right)} \\
=88.34 \cdot \mathrm{N} \frac{\mathrm{s}}{\mathrm{m}} .
\end{array}
$$

At this point, the whole set of fundamental dynamic parameters of the Jeffcott-model corresponding to the real world multi-stage rotor has been identified. However, the question of the unbalance force direction remains still unanswered. Such direction can be specified by Eq. (32) according to

$$
\tan \left(\varphi_{0}\right)=\frac{2 \zeta q_{0}}{1-q_{0}^{2}}=\frac{2 \cdot 0.335 \cdot \frac{32.96}{33}}{1-\left[\frac{32.96}{33}\right]^{2}}=1.0069 \cdot 10^{4} ;
$$

this equation leads to a value for $\varphi_{0}$ of around $88^{0}$. Thus, to produce a well suited balancing force at such resonant rotating velocity it should nearly have the opposite direction as shows Fig. 9. Anyway, the balancing procedure has been exhaustively addressed in textbooks on the subject. ${ }^{14}$ The customary approach establishes the balancing force direction by setting up a vector from the beginning to the end of the resonant hump, as shown in Fig. 13 (keeping in mind the angular reference of the physical rotor). As can be see both directions, the one depicted in Fig. 9 and the second one shown in Fig. 13 agree. Also, this univocal direction has demonstrated to be effective regarding the balancing purposes as corroborates the Fig. 14. This fact reinforces the notion that the results obtained by the presented method agree with the previously mentioned literature on the subject.

\subsection{Keyed shaft rotor}

For the case of a keyed rotor referring to Fig. 15, the orbit has two humps showing a more complex dynamic response. During the whole revolution, the keyed shaft stiffness passes through two cycles, as shows the Fig. 16. Let it be the minimum value $\mathrm{k}-\Delta k$, and the maximum $\mathrm{k}+\Delta k$, with and average value of $k$. Then for uniform rotating speed the instantaneous stiffness can be expressed by

$$
k(t)=k+\Delta k \cdot \sin (2 \omega t) .
$$

The variable component of the spring force becomes a disturbing force of frequency $2 \omega$. If the shaft is running at its half

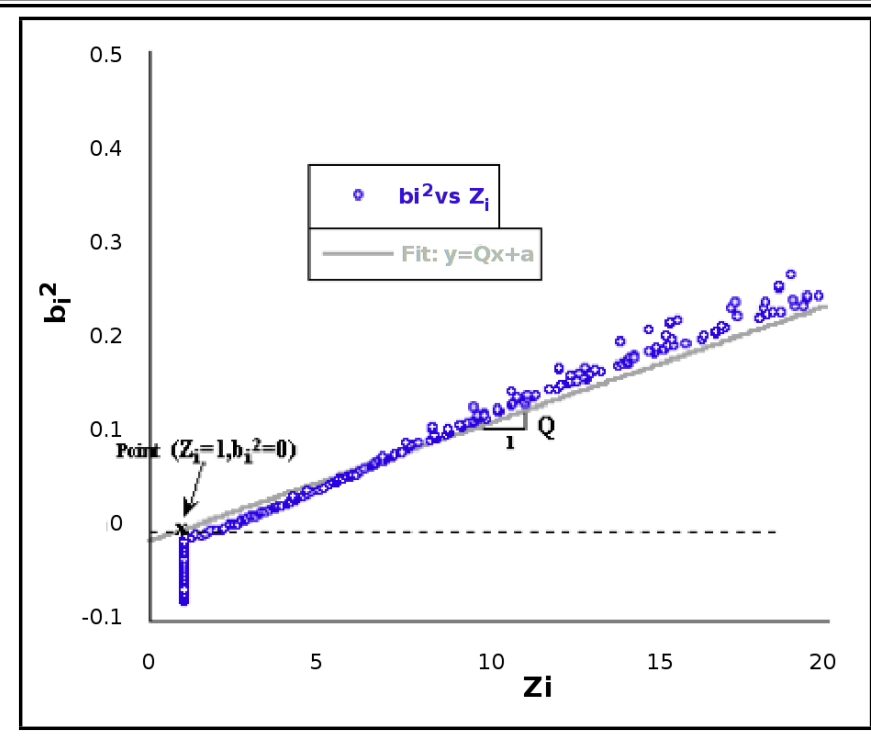

Figure 11. Estimation of the actual critical frequency. Conditions: slope $Q=0.004616 \pm 8.35 \cdot 10^{-5}$.

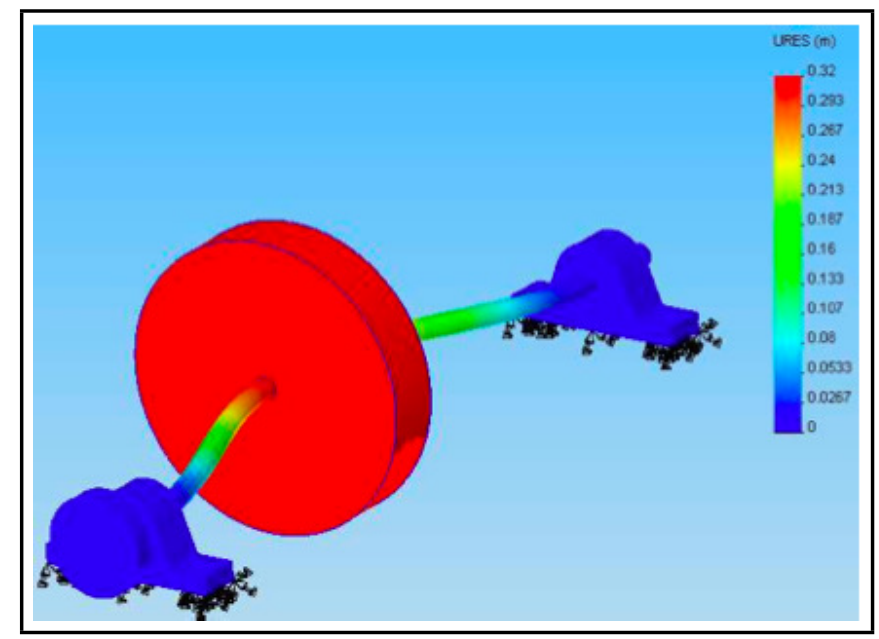

Figure 12. Estimation of the assumed modeled frequency by the Finite Element Method. Result: $\omega_{m} \simeq 44 \mathrm{~Hz}$.

critical speed $\frac{\omega_{0}}{2}$, the impulses of this force will occur at the rotating machine natural frequency. If this occurs, the case a resonant situation occurs delivering a hump into the whole orbit at such frequency: $\omega=\frac{\omega_{0}}{2}$. Thus, a two humps orbit is expected. The first hump occurs closed to the half critical speed plus the second one at the first critical. Both appear in the experimental orbit depicted in Fig. 15. The main hump with constant radius seems to be a circle. The reason can be found in the saturation of the analog probes as a consequence of the vibration severity. This is a weak point of the proposed method. As the pollution of the $R_{i}$ experimental measurements increases, the reliability of the method obviously decreases. furthermore, the translation of the two humps orbit to the $Z_{i}-b_{i}^{2}$ plane is given in Fig. 17 reinforcing the notion of a complex dynamic behavior. Also, Table 4 was built using the same experimental measured data.

After the fitting procedure, the guess frequency corresponding to the linear polynomial, traversing the constraint point $(1,0)$ of the $Z_{i}-b_{i}^{2}$ plane, is $\omega_{m}=26.5$, such polynomial have a slope $Q$ of 0.1772 . Finally, the actual natural frequency found was

$$
\omega_{0}=26.5 \frac{1}{\sqrt[4]{1+0.1772}}=25.44 \mathrm{~Hz} .
$$




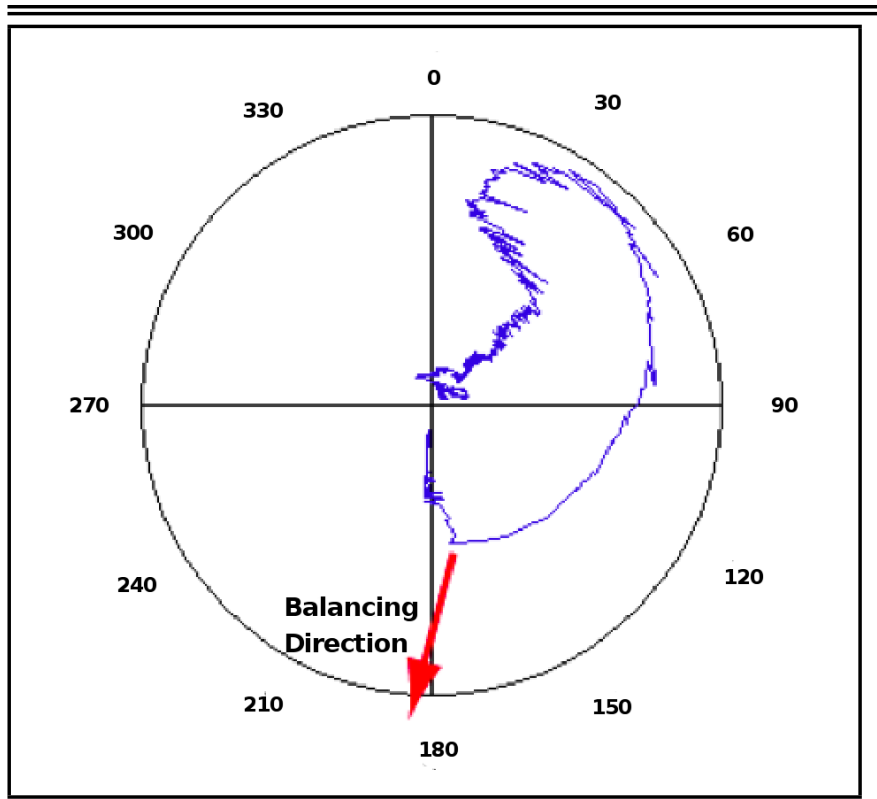

Figure 13. Balancing direction estimation, by setting up a vector from the beginning to the end of the orbit hump. Conditions: clock wise rotation.

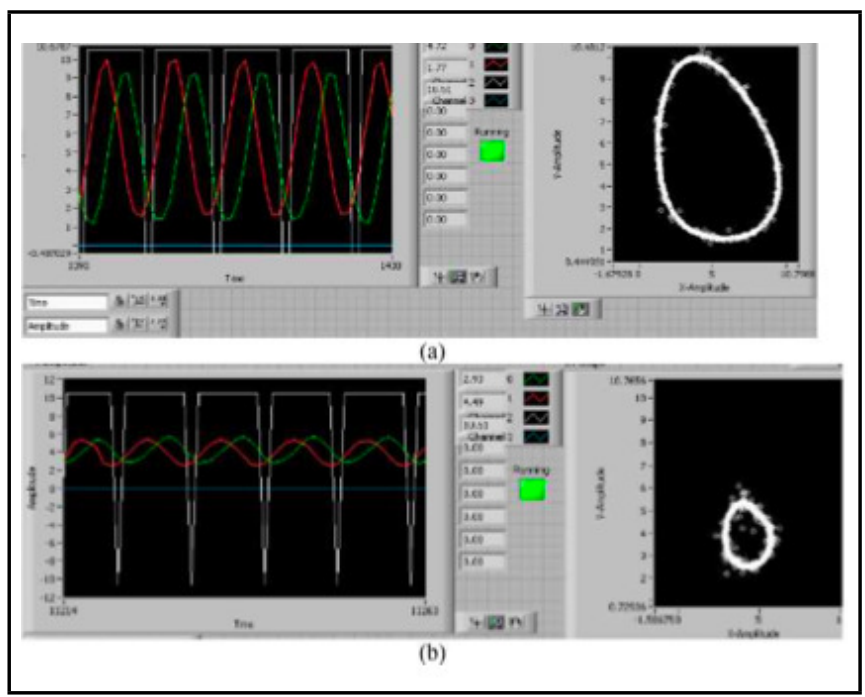

Figure 14. Time-based dynamic signals and polar plot: (a) unbalanced rotor and (b) balanced rotor (two correction weights added). Conditions: mechanical frequency $20 \mathrm{~Hz}$, discrete correction weight $8 \mathrm{gr}$ at the inertial disk surface.

The uncertainty is not important here due to the polluted measurement data. However, in spite of something corrupted data, as expected from a qualitative view point, the global rotating machine natural frequency is shifted down this is caused by the stiffness loose of the keyed shaft if compared with the continuous shaft without the slot along it. This statement can be proved by applying the Eq. (29) to the keyed shaft to estimate the corresponding Jeffcot-model stiffness

$$
\begin{array}{r}
k=\omega_{0}^{2} m \sqrt{1+Q}=(25.44 \cdot 2 \pi)^{2} \\
\cdot 9 \cdot \sqrt{1+0.1772} \\
=2.49 \cdot 10^{5} \mathrm{~N} / \mathrm{m} .
\end{array}
$$

This is a lower value than the prior case of the non-keyed shaft, verifying the expected theoretical result, and reinforces the presented method conformity with the experimental facts.

\section{CONCLUSIONS}

At this point, a Jeffcott-model algebraic method that makes affordable analytical expressions available for engineers

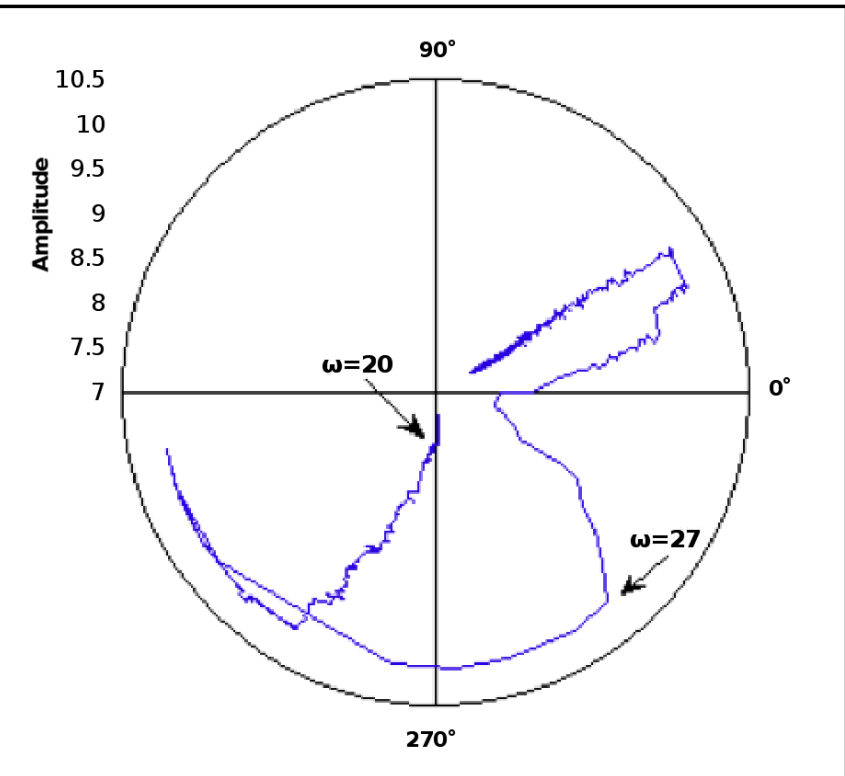

Figure 15. Experimental keyed rotor orbit that was polluted by probes saturation, including one hump of constant radius.

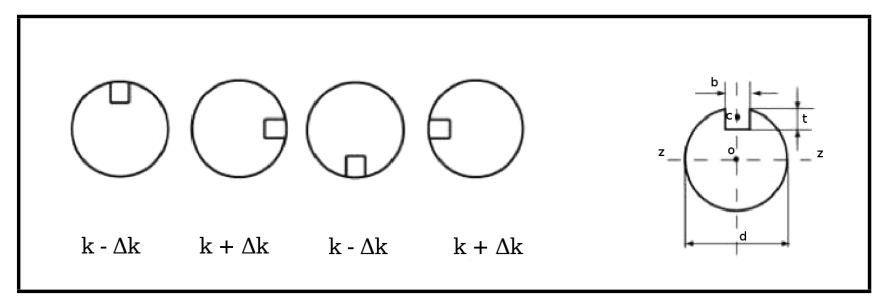

Figure 16. Variable stiffness of the rotor induced by the slot along its length Conditions: $b=5 \mathrm{~mm}, t=2.65 \mathrm{~mm}, d=15 \mathrm{~mm} . \mathrm{Iz}=2485-331.25 \cdot 10^{-12} \mathrm{~m}^{4}$

Table 4. Convergence of the LQE linear polynomial fit procedure for the keyed-rotor.

\begin{tabular}{|l|l|r|r|}
\hline$\omega_{m}$ & linear polynomial & $Z_{i}$ & $b_{i}^{2}$ \\
\hline$\omega_{m}=25$ & $b_{i}^{2}=0.1991 Z_{i}-0.07652$ & 1 & 0.12258 \\
\hline$\omega_{m}=26$ & $b_{i}^{2}=0.1841 Z_{i}-0.1462$ & 1 & 0.0379 \\
\hline$\omega_{m}=26.5$ & $b_{i}^{2}=0.1772 Z_{i}-0.1781$ & 1 & $\gg 0.0009$ \\
\hline
\end{tabular}

to estimate the most descriptive parameters for uncertain dynamics rotating machinery has been developed. Thus, the critical frequency, the stiffness of the shaft, the damping ratio plus the unbalance force phase, are estimated in a straight forward manner on the basis of the correlation between any experimental real-world rotating machine orbit's hump to the Jeffcott-orbit. This comprises an affordable Least Square polynomial fitting procedure applied to the log of to the experimental orbit. The results show clearly the conformity with the physical evidence and accuracy for the aforementioned parameters estimation.

\section{ACKNOWLEDGEMENTS}

The authors wish to acknowledge the financial support of the Spanish government through Ministerio de Ciencia e Innovación and FEDER Funds. Research project ENE2007-6803C04-01 plus research project MAQ-STATUS DPI2015-69325C2-1-R (MINECO/FEDER, UE). 


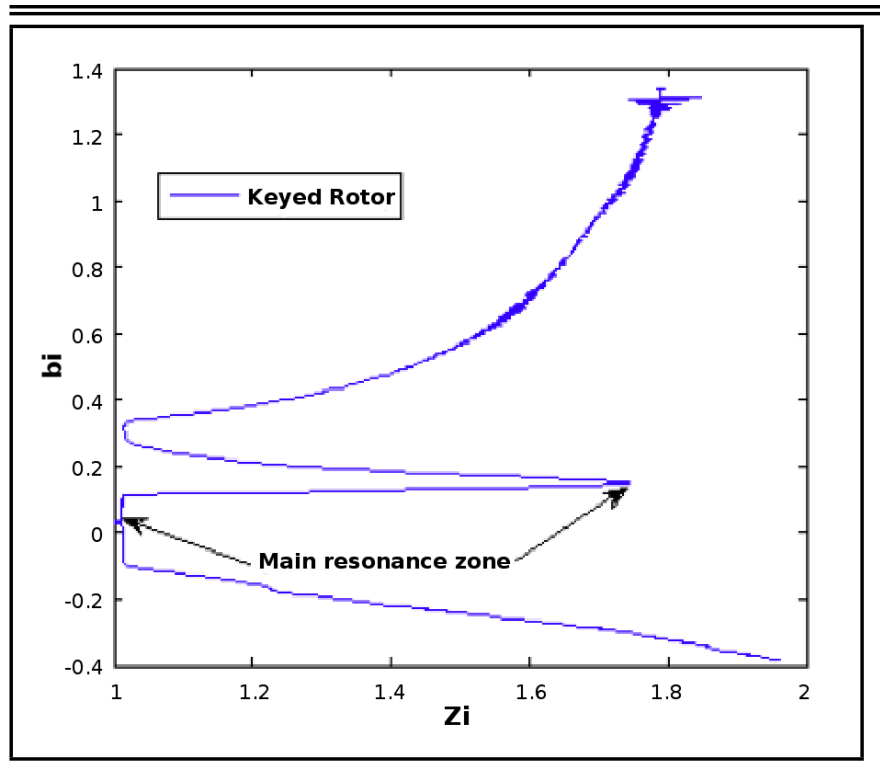

Figure 17. Location of the main resonant zone in the $Z_{i}-b_{i}^{2}$ plane for the keyed rotor.

\section{REFERENCES}

1 Frith, J. and Lamb, E. H. The breaking of shafts in directcoupled units, due to oscillations set up at critical speeds, Journal of the Institution of Electrical Engineers, 31(155), 646-667, (1902). https://dx.doi.org/10.1016/b978-085461-076-1.50025-1

2 Rankine, W. J. Centrifugal whirling of shafts, Engineer, 27, 249, (1869).

3 Smil, V. Creating the Twentieth Century: Technical Innovations of 1867-1914 and Their Lasting Impact, Oxford University Press, (2005).

4 Jeffcott, H. H. The lateral Vibration of Loaded Shafts in the Neighbourhood of a Whirling Speed. The effect of want of balance, Phil. Mag. series 6, 37(219), 304-314, (1919). https://dx.doi.org/10.1080/14786440308635889

5 Grammel, R. Kritische Drehzahl und Kreiselwirkung. (Critical speed and gyroscope effect.), Z.Ver.dtsch. Ing., (44), (1920).

6 Kimball, A. L. Internal friction theory of shaft whipping, Gen. Elec. Rev., 200-244, (1924).

7 Newkirk, B. L. Shaft whipping, Gen. Elec. Rev., 27, 169, (1924)

8 Stodola, A. Dampf und Gasturbinen. (Steam and gas turbines.), Julius Springer, Berlin, (1924).

9 Den Hartog, J. P. Mechanical Vibrations, MacGraw-Hill 4th ed. New York, (1956). https://dx.doi.org/10.1017/s0368393100131049

10 Dick, J. The whirling of shafts having sections with unequal principal bending moduli, Phil. Mag., 299, (1948). https://dx.doi.org/10.1080/14786444808521703

11 Dimentberg, F. M. Flexural Vibrations of Rotating Shafts., Production Engineering Research Association Dept of Scientific and Industrial Research, (1961). https://dx.doi.org/10.1017/s0368393100076331
12 Bently, D. E. Polar plotting Applications for Rotating Machinery, Proceedings of the Vibration Institute Machinery Vibrations IV Seminar, Cherry Hill New Jersey, (1980).

13 Eisenmann, R. C. I-Machinery Monitoring, II-Machinery Vibration., Prentice Hall Inc, (1970).

14 Eisenmann, R. C. Sr. and Eisenmann, R. C. Jr. Machinery Malfunction Diagnosis and Correction, Prentice Hall Inc, (1998).

15 Gasch, R. Dynamic behaviour of the Laval rotor with a transverse crack, Mechanical Systems and Signal Processing, 22, 790-804, (2008). https://dx.doi.org/10.1016/j.ymssp.2007.11.023

16 Nieto, A. J., Morales, A. L., Chicharro, J. M., and Pintado, P. Unbalanced machinery vibration isolation with a semi-active pneumatic suspension. Journal of Sound and Vibration, 329, 3-12, (2010). https://dx.doi.org/10.1016/j.jsv.2009.09.001

17 Cheng, C. C., Wu, F. T., Hsu, K. S., and Ho, K. L. Design and analysis of auto-balancer of an optical disk drive using speed-dependent vibration absorbers. Journal of Sound and Vibration, 311, 200-211, (2008). https://dx.doi.org/10.1016/j.jsv.2007.08.038

18 Chiba, A., Fukao, T., and Rahman, M. A. Vibration Suppression of a Flexible Shaft With a Simplified Bearingless Induction Motor Drive. IEEE Transactions on Industry Applications, 44(3), 745-752, (2008). https://dx.doi.org/10.1109/tia.2008.921401

19 Yong-Chen, P., Lu, H., and Chatwin, C. Dynamics of a rotating shaft-disc under a periodic axial force. Proc. Inst. Mech. Eng. Part $K$ Journal of Multi-body Dynamics., 2, 211-219, (2010). https://dx.doi.org/10.1243/14644193jmbd209

20 Sorge, F. and Cammalleri, M. Control of hysteretic instability in rotating machinery by elastic suspension systems subject to dry and viscous friction. Journal of Sound and Vibration, 329(10), 1686-1701, (2010). https://dx.doi.org/10.1016/j.jsv.2009.12.007

21 Verichev, N. N., Verichev, S. N., and Erofeyev, V. I. Damping lateral vibrations in rotary machinery using motor speed modulation. Journal of Sound and Vibration, 329, 13-20, (2010). https://dx.doi.org/10.1016/j.jsv.2009.09.018

22 Meirovitch, L. Fundamentals of Vibrations. McGraw-Hill International Edition, (2001). https://dx.doi.org/10.1115/1.1421112

23 Peláez G., Caparrini N., and García-Prada J. C. Two impulse sequence input shaper design for link mechanism with non-linearities. Proceedings of the American Control Conference, New York (2007). https://dx.doi.org/10.1109/acc.2007.4282326

24 Graham Kelly S. Mechanical Vibrations. MacGraw-Hill Shaum's OutLines, (1996). 